\title{
Critical Fidelity at the Metal-Insulator Transition
}

\author{
Gim Seng Ng ${ }^{1,2}$, Joshua Bodyfelt ${ }^{1}$ and Tsampikos Kottos $^{1,2}$ \\ ${ }^{1}$ Department of Physics, Wesleyan University, Middletown, Connecticut 06459, USA \\ ${ }^{2}$ Max-Planck-Institute for Dynamics and Self-Organization, \\ Bunsenstraße 10, D-37073 Göttingen, Germany
}

\begin{abstract}
Using a Wigner Lorentzian Random Matrix ensemble, we study the fidelity, $F(t)$, of systems at the Anderson metal-insulator transition, subject to small perturbations that preserve the criticality. We find that there are three decay regimes as perturbation strength increases: the first two are associated with a gaussian and an exponential decay respectively and can be described using Linear Response Theory. For stronger perturbations $F(t)$ decays algebraically as $F(t) \sim t^{-D_{2}^{\mu}}$, where $D_{2}^{\mu}$ is the correlation dimension of the Local Density of States.
\end{abstract}

PACS numbers:

The theory of fidelity [1] (also known as Loschmidt Echo) has been a subject of intensive research activity during the last years (for a recent review see [2]). This interest has been motivated by various areas of physics, ranging from atomic optics 3, 4, 5], microwaves [6] and elastic waves $7 \mid$ to quantum information $[8]$ and quantum chaos [9, 10, 11, 12, 13, 14, 15, 16, 17, 18]. It has been adopted as a standard measure for quantum reversibility and stability of quantum motion with respect to changes in an external parameter $x_{\mathrm{e}}$. Formally, the fidelity $F(t)$, is defined as:

$$
F(t)=\left|\left\langle\psi_{0}\left|\mathrm{e}^{i H_{\mathrm{b}} t} \mathrm{e}^{-i H_{\mathrm{f}} t}\right| \psi_{0}\right\rangle\right|^{2} ; \quad \hbar=1
$$

where $\mathbf{H}_{\mathrm{f}}$ and $\mathbf{H}_{\mathrm{b}}=\mathbf{H}_{\mathrm{f}}+x_{\mathrm{e}} \mathbf{B}$ represent the reference Hamiltonian and its perturbed variant, respectively, while $\left|\psi_{0}\right\rangle$ is an initial state. One can interpret fidelity (11) in two equivalent ways. It can be considered as the overlap of an initial state with the state obtained after the forward unperturbed evolution, followed by a backward perturbed evolution. Equivalently, it is the overlap of a state obtained after a forward unperturbed evolution and the state after a forward perturbed evolution. The latter interpretation is closely linked to the concept of dephasing [19] in mesoscopic devices and coherent manipulation of a quantum state. Sustaining the coherence of a superposition of state vectors is at the heart of quantum parallelism in quantum computation schemes [8, 20, 21]. The first interpretation goes back to the original proposal by Peres [1], who used fidelity to study quantum-classical correspondence and identify traces of classical (chaotic or integrable) dynamics in quantized systems.

For a quantum system with a classical chaotic counterpart, the decay of the fidelity depends on the strength of the perturbation parameter $x_{\mathrm{e}}$. Recent studies indicated that there are three $x_{\mathrm{e}}$-regimes: the standard perturbative regime, the Fermi Golden Rule regime (FGR), and the non-perturbative regime. The first two can be described by Linear Response Theory (LRT) leading to a decay which depends on the perturbation strength $x_{\mathrm{e}}$ as $F(t) \sim \mathrm{e}^{-\left(x_{\mathrm{e}} t\right)^{2}}$ and $F(t) \sim \mathrm{e}^{-x_{\mathrm{e}}^{2} t}$, respectively [10, [13]. In the non-perturbative regime, the decay is $F(t) \sim \mathrm{e}^{-\lambda t}$, with a rate that is perturbation independent and is given by the Lyapunov exponent $\lambda$ of the underlying classical system [9, 10, 18].

The investigation of the fidelity has recently been extended to systems that have integrable classical dynamics. It was shown 11] that the decay follows a power law $F(t) \sim t^{-3 d / 2}$, where $d$ is the dimensionality of the system. A similar algebraic decay was found for disordered systems with diffractive scatterers, where now the power law is governed by the diffusive dynamics [22].

Despite the progress in understanding the fidelity of various systems, a significant class was left out of the investigation. These are systems which show an Anderson metal-insulator transition (MIT) as an external parameter changes. In the metallic regime, the eigenstates of these systems are extended, and the statistical properties of their spectrum are quite well described by random matrix theory [23]. In particular, the level spacing distribution is very well fitted by the Wigner surmise. Deep in the localized regime, the levels become uncorrelated leading to a Poissonian level spacing distribution and the eigenfunctions are exponentially localized. At the MIT, the eigenfunctions are critical, exhibiting multifractal structure characterized by strong fluctuations on all scales. The eigenvalue statistics are characterized by a third universal distribution [23, 24]. Representatives of this class are disordered systems in $d>2$ dimensions, two-dimensional systems in strong magnetic fields (quantum Hall transition), or periodically kicked systems with a logarithmic potential singularity [25].

Here, for the first time, we address the behavior of $F(t)$ for systems at criticality and present consequences of the MIT on the fidelity decay. Using the Wigner Lorentzian Random Matrix (WLRM) ensemble, we find that there are three regimes: (a) the standard perturbative regime where the decay is gaussian; (b) the FGR decay where the decay is exponential and (c) the non-perturbative regime where an initial gaussian decay (Zeno decay) is followed by a power law. The latter decay is novel and reflects the 
critical nature of the system. Specifically we found that

$$
F(t) \sim \frac{1}{t^{D_{2}^{\mu}}}
$$

where $D_{2}^{\mu}=D_{2}^{\psi} / d$ [26] is the correlation dimension of the Local Density of States (LDoS) while $D_{2}^{\psi}$ is the correlation dimension of the critical eigenstates and $d$ is the actual dimensionality of the system. For the WLRM model $D_{2}^{\mu}=D_{2}^{\psi}=D_{2}$ since $d=1$. The correlation dimension $D_{2}^{\psi}$ is usually defined through the inverse participation ratio, $P_{2}=\int d^{d} r|\psi(r)|^{4} \sim L^{-D_{2}^{\psi}}$, where $L$ is the size of the system [27]. The correlation dimension is also related to the spectral compressibility $\chi=\left(d-D_{2}^{\psi}\right) / 2 d$, defined through the level number variance $(\delta N)^{2} \approx \chi\langle N\rangle$ [28, 29, 30]. At the same time, $D_{2}^{\psi}$ manifests itself in a variety of other physical observables. As examples, we mention the conductance distribution [31, 32], the anomalous spreading of a wave-packet 33], the spatial dispersion of the diffusion coefficient [34, 35, 36], and the anomalous scaling of Wigner delay times 37.

We use the WLRM model [38, 39, 40], defined as:

$$
\mathbf{H}=\mathbf{H}_{\mathbf{0}}+x \mathbf{B}
$$

Both $\mathbf{H}_{\mathbf{0}}$ and $\mathbf{B}$ are real symmetric matrices of size $L \times$ $L$ with matrix elements randomly drawn from a normal distribution with zero mean and a variance depending on the distance of the matrix element from the diagonal

$$
\left\langle\sigma_{n m}^{2}\right\rangle=\frac{1}{1+\left|\frac{n-m}{b}\right|^{2}} .
$$

where $b \in(0, L)$ is a free parameter that controls the critical properties of the system (see Eq. (5) below). Random matrix models with variance given by (4) were introduced in [38] and further studied in 29, 39, 41, 42]. Field-theoretical considerations [38, 39, 41] and detail numerical investigations [29, 42] verify that the model shows all the key features of the Anderson MIT, including multifractality of eigenfunctions and non-trivial spectral statistics at the critical point. A theoretical estimation for the correlation dimension $D_{2}^{\psi}$ gives [38]

$$
D_{2}^{\psi}=\left\{\begin{array}{cc}
4 b \Gamma(3 / 2)[\sqrt{\pi} \Gamma(1)]^{-1} & , b \ll 1 \\
1-2(2 \pi b)^{-1} & , b \gg 1
\end{array}\right.
$$

where $\Gamma$ is the Gamma function.

The forward and backward Hamiltonians used for the calculation of the fidelity (11) are [43]

$$
\mathbf{H}_{\mathrm{f}}=\mathbf{H}(x) \quad \text { and } \quad \mathbf{H}_{\mathrm{b}}=\mathbf{H}(-x)
$$

We operate in the basis where $\mathbf{H}_{\mathbf{0}}$ is diagonal [40]. In this basis, the perturbation matrix $\mathbf{B}$ is $x$-invariant [40], i.e. it preserves the same Lorentzian power-law shape (4), while its critical properties (like the multifractal dimension $D_{2}^{\psi}$ ) remain unchanged. For the numerical evaluation of $F(t)$, we have used two types of initial conditions

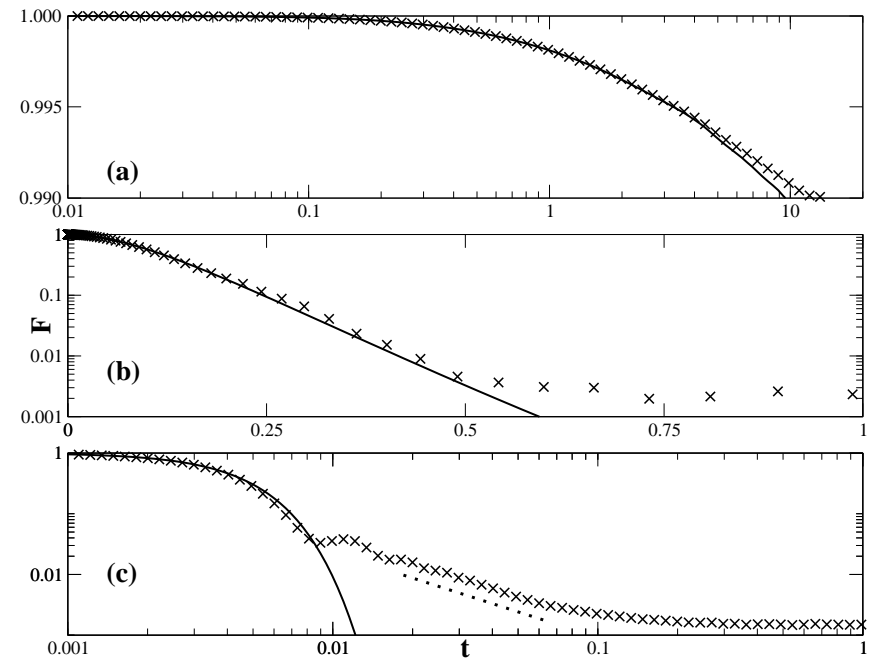

FIG. 1: Fidelity of an ES, for (a) $x=0.01$ (the standard perturbative regime), (b) $x=0.8$ (FGR regime) and (c) $x=20$ (non-perturbative regime). The solid lines are the LRT results from Eq. (7) while the crosses are the outcomes of the numerical simulations with model (3, 4). In these simulations $L=1000$ and $b=10$. The mean level spacing of the unperturbed system is set to $\Delta \approx 1$. In this case, $x_{c} \approx 0.59$ and $x_{\text {prt }} \approx 1.88$. The dotted line in $(\mathrm{c})$ is plotted to guide the eye on the power-law behavior.

$\left|\psi_{0}\right\rangle$ : an eigenstate of $\mathbf{H}_{\mathbf{0}}$ (ES) and a generic "random" state (RS). In both cases, the results are qualitatively the same. Therefore, we will not distinguish between them. In our numerical experiments we used matrices of size varying from $L=1000$ to $L=5000$. We have performed an averaging over different initial states and realizations of the perturbation matrix $\mathbf{B}$ (typically more than 1000).

An overview of the temporal behavior of the fidelity $F(t)$ for three representative perturbation strengths is shown in Fig. 1. For perturbation strengths smaller than $x_{c} \approx \frac{\Delta}{\sqrt{\pi}} \sqrt{1+\frac{1}{b}}$ [44], the decay of $F(t)$ is gaussian (see Fig. 1a). The perturbative border $x_{c}$ is the perturbation strength needed in order to mix levels within a distance of a mean level spacing $\Delta$ [40]. Above this border, one typically expects an exponential FGR decay of fidelity [10], with a rate given by the width of the Local Density of States (LDoS) [40] (see Fig. 1b). We can apply LRT [2] to evaluate the decay of $F(t)$ in these two regimes. The resulting expression reads

$$
\langle F(t)\rangle_{B, n_{0}} \approx 1-(2 x)^{2} \mathcal{C}(t) \approx \mathrm{e}^{-(2 x)^{2} \mathcal{C}(t)}
$$

where $\langle\ldots\rangle_{B, n_{0}}$ represents a double average over $\mathbf{B}$ and initial states. The right hand side of expression (7) assumes the validity of infinite order perturbation theory. The correlator $\mathcal{C}(t)$ is

$$
\mathcal{C}(t)=\int_{0}^{t} d \tau_{1} \int_{0}^{\tau_{1}} d \tau_{2} \sum_{n}\left|c_{n}\right|^{2} \tilde{C}_{n}\left(\tau_{1}-\tau_{2}\right)-2 \mathcal{I} t^{2}
$$




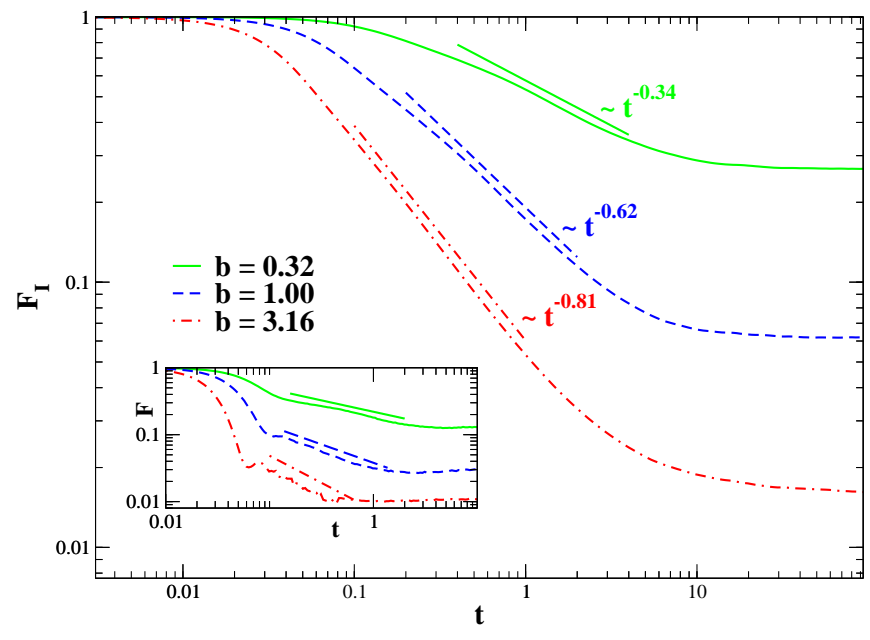

FIG. 2: (color) $F_{I}$ for $b=0.32,1.00$ and 3.16. The initial state was chosen to be an ES. The mean level spacing of the unperturbed Hamiltonian is set to be $\Delta \approx 1$, while the size of the matrices is $L=5000$. In the cases reported here we have choose $x=5$. In the inset, we also present the fidelity for RS for $b=0.32$ and $b=1.00$ using the same parameters except $L=1000$. The straight lines are plotted to guide the eye.

where $\mathcal{I}=\sum_{n}\left|c_{n}\right|^{4}$ is the inverse participation ratio of the initial state, $\tilde{C}_{n}\left(t-t^{\prime}\right) \equiv 2\left(1+\sum_{\gamma} \sigma_{n, \gamma}^{2} \cos \left[\left(E_{\gamma}^{(0)}-\right.\right.\right.$ $\left.\left.\left.E_{n}^{(0)}\right)\left(t-t^{\prime}\right)\right]\right)$, and $E_{n}^{(0)}$ denotes an eigenvalue of $\mathbf{H}_{\mathbf{0}}$. In the case of standard GOE ensembles with $\sigma_{n m}^{2}=1$, Eq. (77) reduces to the expression derived in [13]. The prediction of LRT (17) is plotted together with the numerical results in Fig. 1 for different perturbation strengths. A good agreement between Eq. (7) and the numerical data is observed for perturbation strengths less than $x_{\mathrm{prt}} \approx \Delta \sqrt{b} \frac{\sqrt{\pi-1.28[\pi / 2-\arctan (1 / b)]}}{1.68[\pi / 2-\arctan (1 / b)]}$ (see Figs. 1a,b) [44].

For $x$ larger than $x_{\mathrm{prt}}$ the decay of $F(t)$ cannot be captured by LRT (see Fig. 1c). The non-perturbative character of this regime was identified already in the frame of the parametric evolution of the Local Density of States (LDoS) [40]. A representative temporal behavior of $F(t)$ for $x>x_{\text {prt }}$ is reported in Fig. 1c. For short times the decay of $F(t)$ is gaussian. For longer times, we can observe a transition to a power law decay. The initial gaussian decay $F(t) \sim \mathrm{e}^{-x^{2} t^{2}}$ is universal and can be identified with the quantum Zeno effect [1, 2]. It is valid until times $t_{Z} \sim 1 / x$. We will focus in the observed power-law decay which take place for $t>t_{Z}$.

The numerical results for three different b-values, $b=$ $0.32,1$, and 3.16, are reported in Fig. 2. We use the time-averaged fidelity

$$
F_{I}(t) \equiv\langle F(t)\rangle_{t}=\frac{1}{t} \int_{0}^{t} F(t) d t
$$

to reduce further statistical fluctuations. In the inset, we present the raw data for the fidelity decay. In all cases the fidelity $F(t)$ clearly displays an inverse power law,

$$
F(t) \propto \frac{1}{t^{\gamma}}
$$

with a power $\gamma$ that depends on the band-width parameter $b$. By fitting our data to Eq. (10), the power-law exponent $\gamma$ is extracted. In Fig. 3 we summarize the extracted $\gamma$ 's for both ES and RS initial conditions as a function of the bandwidth $b$. The results are essentially identical within the numerical accuracy of our fitting procedure.

If the initial state $\left|\psi_{0}\right\rangle$ is an eigenstate of the backward (or forward) Hamiltonian then the fidelity is simply the survival probability $P(t) \equiv\left|\left\langle\psi_{0}\left|\mathrm{e}^{-i H_{\mathrm{f}} t}\right| \psi_{0}\right\rangle\right|^{2}$ of wavepacket dynamics. In the latter case, it is known that the survival probability at criticality decays as $P(t) \sim 1 / t^{D_{2}^{\mu}}$ 33]. However, in these fidelity experiments, the initial state is neither eigenstate of $H_{\mathrm{b}}$ nor of $H_{\mathrm{f}}$. In fact, Ref. [15] shows that the physics of quantum fidelity involves subtle cross correlations which in general are not captured by the survival probability (or the LDoS which is its Fourier transform) alone. Motivated by this equivalence between fidelity and survival probability for the specific choice of initial condition $\left|\psi_{0}\right\rangle$, we have compared in Fig. 3 the extracted power law exponents $\gamma$ with the correlation dimension $D_{2}^{\mu}=D_{2}^{\psi}=D_{2}$ [42]. The agreement between the $\gamma$ and the $D_{2}^{\mu}$ is excellent for all $b^{\prime} \mathrm{s}$ confirming the prediction (2).

The connection between the exponent $\gamma$ and the fractal dimension $D_{2}^{\mu}$ calls for an argument for its explanation. The following heuristic argument provides some understanding of the power law decay Eq. (2). For any finite Hilbert space the fidelity $F(t)$ approaches the value $F_{\infty}=1 / L$, being the inverse of the dimension of the Hilbert space. If the dynamics, however, take place in a space with an effective reduced dimension $D_{2}^{\psi}$, we will have $F_{\infty}=1 / L^{D_{2}^{\psi}}$ [46]. Assuming a power law decay (10) for the fidelity, we can estimate how the time $t_{*}$ at which $F\left(t_{*}\right)=F_{\infty}$ scales with $L$, i.e. $t_{*} \sim L^{D_{2}^{\psi} / \gamma}$. On the other hand, the dynamics of a critical system is characterized by an anomalous diffusive law $L^{2} \sim t_{*}^{2 D_{2}^{\mu} / D_{2}^{\psi}}$ [33] which defines the time $t_{*} \sim L^{D_{2}^{\psi} / D_{2}^{\mu}}$ needed to explore the available space $L$. Equating the two expressions for $t_{*}$ we finally get that $\gamma=D_{2}^{\mu}$. Although the numerical results leaves no doubt on the validity of Eq. (2), a rigorous mathematical proof is more than desirable.

In conclusion, we have investigated the fidelity decay for systems at MIT. Depending on the perturbation strength $x$, we have indentified three distinct regimes: For $x<x_{c}$ the fidelity decay is gaussian; for $x_{c}<x<$ $x_{\text {prt }}$ the decay is exponential and for $x>x_{\text {prt }}$ the decay is power law. The first two regimes are described by LRT. The third is non-perturbative. The power law decay is dictated by the critical nature of the system. Specifically, we have found that the power-law exponent is equal to 


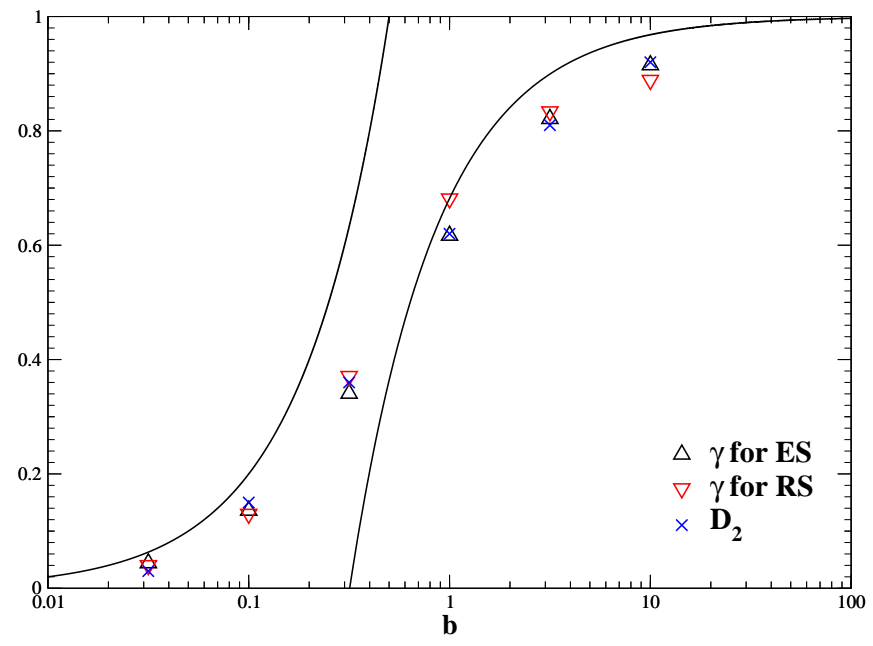

FIG. 3: (color) The fitting parameter $\gamma$ for $b=$ $0.03,0.10,0.32,1.00,3.16$, and 10 . We are using $\Delta=1, x=5$. The analytical (solid lines) Eq. (5) and numerical (crosses) [45] results for $D_{2}$ are also shown for comparison.

the correlation dimension of the critical eigenstates.

We acknowledge T. Geisel for his continuous interest and support of this project. Useful discussions with D. Cohen, T. Gorin, M. Hiller, S. Coppage, and A. MendezBermudez are also acknowledged.

[1] A. Peres, Phys. Rev. A 30, 1610 (1984).

[2] T. Gorin, T. Prosen, T. H. Seligman, M. Znidaric, quant-ph/0607050 (2006).

[3] S. A. Gardiner, J. I. Cirac and P. Zoller, Phys. Rev. Lett. 79, 4790 (1997).

[4] M. F. Andersen, A. Kaplan and N. Davidson, Phys. Rev. A 64, 043801 (2001).

[5] S. Kuhr et al. Phys. Rev. Lett. 91, 213002 (2003).

[6] R. Schafer et al., New J. Phys. 7, 152 (2005).

[7] O. Lobkis and R. Weaver, Phys. Rev. Lett. 90, 254302 (2003).

[8] M. A. Nielsen and I. L. Chuang, Quantum computation and quantum information (Cambridge University Press, 2000).

[9] R. A. Jalabert and H. M. Pastawski, Phys. Rev. Lett. 86, 2490 (2001); F. M. Cucchietti, H. M. Pastawski and R. Jalabert, Physica A, 283, 285 (2000); F. M. Cucchietti, H. M. Pastawski and D. A. Wisniacki, Phys. Rev. E 65, 046209 (2002).

[10] Ph. Jacquod, I. Adagdeli and C. W. J. Beenakker, Phys. Rev. Lett. 89, 154103 (2002); Ph. Jacquod, P. G. Silvestrov and C. W. J. Beenakker, Phys. Rev. E 64, 055203(R) (2001).

[11] P. Jacquod, I. Adagideli and C. W. J. Beenakker, Europhys. Lett.61, 729 (2003).

[12] N. R. Cerruti and S. Tomsovic, Phys. Rev. Lett. 88, 054103 (2002); J. Phys. A: Math. Gen. 36, 3451 (2003).

[13] T. Gorin, T. Prosen, T. H. Seligman, New J. Phys. 6, 1 (2004); T. Prosen, Phys. Rev. E 65, 036208 (2002);
T. Prosen and M. Znidaric, J. Phys. A: Math. Gen. 35, 1455 (2002); ibid 34, L681 (2001); T. Prosen and T. H. Seligman, ibid 35, 4707 (2002); T. Kottos and D. Cohen, Europhys. Lett. 61, 431 (2003).

[14] G. Benenti and G. Casati, Phys. Rev. E 66, 066205 (2002); W. Wang and B. Li, ibid. 66, 056208 (2002).

[15] D. A. Wisniacki and D. Cohen, ibid. 66, 046209 (2002).

[16] H. M. Pastawski, P. R. Levstein and G. Usaj, Phys. Rev. Lett.75, 4310 (1995).

[17] J. Vanicek and Eric J. Heller, quant-ph/0302192

[18] F. M. Cucchietti, C. H. Lewenkopf, E. R. Mucciolo, H. M. Pastawski and R. O.Vallejos, Phys. Rev. E 65, 046209 (2002).

[19] W. H. Zurek, Phys. Today 44, 36 (1991); D. Cohen and T. Kottos Phys. Rev. E 69, 055201(R) (2004); D. Cohen, Phys. Rev. E 65, 026218 (2002).

[20] A. Stern, Y. Aharonov and Y. Imry, Phys. Rev. A 41,3436 (1990).

[21] G. A. Fiete and E. J. Heller, Physical Review A 68, 022112 (2003).

[22] Y. Adamov, I. V. Gornyi and A. D. Mirlin, Phys. Rev. E 67, 056217 (2003).

[23] B. I. Shklovskii, B. Shapiro, B. R. Sears, P. Lambrianides, and H. B. Shore, Phys. Rev. B 47, 11487 (1993)

[24] B. L. Alt'shuler and B. I. Shklovskii, Zh. Eksp. Teor. Fiz. 91, 220 (1986) [ Sov. Phys. JETP 64, 127 (1986)]; D. Braun, G. Montambaux, and M. Pascaud, Phys. Rev. Lett. 81, 1062 (1998).

[25] A. M. Garcia-Garcia, and J. Wang, Phys. Rev. Lett. 94, 244102 (2005).

[26] B. Huckenstein and R. Klesse, Phys. Rev. B 55, R7303 (1997)

[27] F. Wegner, Phys. Rep. 67, 15 (1980).

[28] J. T. Chalker, V. E. Kravtsov and I. V. Lerner, JETP Letters 64, 386 (1996)

[29] F. Evers and A. D. Mirlin, Phys. Rev. Lett. 84, 3690 (2000); E. Cuevas, M. Ortuno, V. Gasparian, and A. Perez-Garrido, ibid. 88, 016401 (2002).

[30] A. D. Mirlin and F. Evers, Phys. Rev. B 62,7920 (2000).

[31] D. Braun, E. Hofstetter, G. Montambaux and A. MacKinnon, Phys. Rev. B 64,155107 (2001).

[32] D. G. Polyakov, Phys. Rev. Lett. 81,4696 (1998).

[33] R. Ketzmerick, K. Kruse, S. Kraut and T. Geisel, Phys. Rev. Lett. 79, 1959 (1997).

[34] J. T. Chalker and G. J. Daniell, Phys. Rev. Lett. 61, 593 (1988).

[35] J. T. Chalker, Physica A 167, 253 (1990).

[36] B. Huckestein and R. Klesse, Phys. Rev. B 59, 9714 (1999).

[37] J. A. Mendez-Bermudez and T. Kottos, Phys. Rev. B $\mathbf{7 2}, 064108(2005)$.

[38] A. D. Mirlin, Y. V. Fyodorov,F.-M. Dittes, J. Quezada and T. H. Seligman, Phys. Rev. E 54, 3221 (1996).

[39] V. E. Kravtsov and K. A. Muttalib, Phys. Rev. Lett. 79, 1913 (1997); V. E. Kravtsov and A. M. Tsvelik, Phys. Rev. B 62, 9888 (2000).

[40] J. A. Mendez-Bermudez, T. Kottos and D. Cohen, Phys. Rev. E 73, 036204 (2006).

[41] A. D. Mirlin, Phys. Rep. 326, 259 (2000).

[42] I. Varga, Phys. Rev. B 66, 094201 (2002); I. Varga and D. Braun, ibid. 61, R11859 (2000).

[43] From Eq. (11) and Eq. (6) we see that $x_{\mathrm{e}}=2 x$.

[44] The values of $x_{c}$ and $x_{\text {prt }}$ were derived in [40] on the basis of the LDoS analysis. 
[45] J. A. Mendez-Bermudez, unpublished (2006).

[46] For $x>x_{\text {prt }}$ the wavefunctions are fractal and therefore dimensionality given by $D_{2}^{\psi}$ [4]. fill only a fraction of the available space with an effective 